\title{
Soft computing and intelligent systems: techniques and applications
}

\author{
Sabu M. Thampi ${ }^{\mathrm{a}, *}$, El-Sayed M. El-Alfy ${ }^{\mathrm{b}}$ and Ljiljana Trajkovic ${ }^{\mathrm{c}}$ \\ ${ }^{\mathrm{a}}$ Indian Institute of Information Technology and Management-Kerala, Technopark Campus, \\ Trivandrum, Kerala, India \\ ${ }^{\mathrm{b}}$ Department of Information and Computer Science, College of Computer Sciences and Engineering, \\ King Fahd University of Petroleum and Minerals, Dhahran, Saudi Arabia \\ ${ }^{\mathrm{c}}$ School of Engineering Science, Simon Fraser University, Vancouver, Canada
}

This special issue is composed of a collection of selected papers presented at the Fifth International Symposium on Intelligent Systems Technologies and Applications (ISTA'19) that was organized in association with Springer, Germany and IOS-Press, Netherlands. ISTA'19 was co-affiliated with the International Conference on Applied Soft computing and Communication Networks (ACN'19), held in Trivandrum, Kerala, India during December 18-21, 2019. The Symposium brought together researchers in related fields and provided a venue to explore and discuss various aspects and recent advances in the area of intelligent systems technologies and applications. It offered an excellent opportunity for the presentation and discussion of interesting new research results, which contributed to effective dissemination and transfer of knowledge.

Included in this special issues are 50 selected papers covering topics pertaining to the application of soft computing and computational intelligence techniques to a wide spectrum of issues in the areas of image processing and computer vision, natural language processing, pattern recognition, biometric identification, security systems and data encryption, cloud computing, control systems, and network design.

\footnotetext{
*Corresponding author. Sabu M. Thampi, Indian Institute of Information Technology and Management-Kerala, Technopark Campus, Trivandrum-695581, Kerala, India. E-mail: sabu.thampi @iiitmk.ac.in.
}

Several papers in this special issue have addressed natural language processing. Contribution [1] describes a technique for solving the word sense disambiguation using combinatorial particle swan optimization. A multi-document summarization technique proposed in [2] is based on the integration of regression and topic-based models to improve the reported results. Sentiment analysis of social media data is an evolving area in natural language processing in recent years. Several machine learning algorithms [3] are compared for imbalanced learning of sentiment polarity as a tool to understand the opinions of participants in social media. An automated genre-based sentiment lexicon is proposed in [4] to address the challenge due to variation in sentiments across multiple domains. Another important related area due to the explosive growth of online information is using recommendation systems to filter information in activities such as watching videos and movies, tourism and traveling, as well as food and restaurants. In [5], the researchers developed a system that combines social factors such as trust and reputation to determine influential users in cross domain group recommender systems.

Another vital area is pattern recognition and visual computing. A computationally and storage efficient method is presented in [6] for recognizing human activities from depth videos. A more accurate, time and cost-effective ensemble machine learning is proposed in [7] for recognition of coal category from 
microscopic images. The identification of landmark features in retinal fundus photograph such as optic disc (OD) is of high prognostic significance in diagnosing various ophthalmic diseases. In [8], a fully convolutional neural network framework is proposed for the localization of optic disc based on the underlying architecture of YOLOv3. Automatic separation and classification of tumors in ultrasound images is difficult due to the noisy and low contrast nature of the image. A proposed evolutionary intelligence method [9] is invariant to scale changes and does not require an operator defined initial region of interest. A layered approach is described in [10] for deploying live video surveillance with a deep learning strategy to detect elephants and mitigate the problem of human-elephant conflict in rural areas of India. A study in [11], proposed to develop a system to support a psychologist to understand the participant performance in a trail making test, which is a cognitive impairment test used for understanding the visual attention during a visual search task. Another problem addressed in this special issue related to scene understanding and exploration is the target search problem using a fuzzy-based system to predict the salient locations in an image for visual attention [12]. The algorithm described in [13] aims to detect localized edges in noisy images without duplicate and integrating them into meaningful object boundaries.

Signal processing and speech analysis are active fields that have received great interest in many applications. In [14], the authors utilized speech cues represented by excitation source features, voice quality features, and prosodic features to identify patients affected by a vital neurodegenerative condition of Parkinson disease (PD) from healthy individuals who have no history of such disorder. Two machine learning classification techniques (random forest and support vector machine) have been evaluated using a Parkinson's database from the University of California Irvine (UCI) Machine Learning repository. A cascade of two deep-learning architecture is proposed in [15] for accent identification. The model has been developed and tested on common voice datasets with five popular English accents: India, Australia, US, England, and Canada. In [16], a random kitchen sink based approach is proposed for music-speech classification using temporal and spectral features achieving scores comparable to other methods reported in the literature. Acquiring and processing brain signals to understand students concentration may have a great impact on education. A case study presented in [17] analyzes the influence of practicing Yoga on the concentration levels of engineering students. Underwater acoustics poses several challenges that attracted increasing attention to address problems such as sensors sensitivity, sound intensity measurement, and target localization. The authors of [18] propose to enhance the fiber optic hydrophone sensitivity by varying parameters as demonstrated through analytical results. Another interesting problem is addressed in [19] where a hybrid architecture that performs multiple transforms of digital signals such as Discrete Fourier Transform (DFT), Discrete Cosine Transform (DCT), Discrete Sine Transform (DST), and Discrete Wavelet Transform (DWT) was proposed. The designed architecture produces output of transform sequence in order and may be used to handle large sized transforms by repeatedly using fixed size architecture for a large number of points without increasing the number of processing elements.

Security and digital forensic represent several challenges that have attracted significant research efforts. With the availability of powerful image editing tools, manipulating images to change their content is becoming a concern. Image integrity and forgery detection are addressed in [20] using deep textural features from Local Binary Pattern (LBP) map. Benchmarking the proposed methodology is reported using four datasets (CASIA v1.0, CASIA v2.0, CUISDE, and IFS-TC). Information hiding in digital images is another important issue in data security. A novel technique with high payload is presented in [21] for hiding confidential messages in encrypted images while enabling separate image recovery and information extraction. The test results show high embedding capacity with $100 \%$ restoration of cover images and extraction of secret information. Biometric identification is addressed in [22, 23]. In [22], the authors propose a deep learning approach for an emerging biometric trait using tongue print identification based on the remarkable success of convolutional neural networks for feature extraction and pattern recognition. A dataset is also created for testing the proposed approach. The approach in [23] targets face recognition in unconstrained environment where images are affected by various types of occlusion. A multi-biometric cryptosystem is presented in [24] for safe and secure cloud authentication mechanism. Outsourcing data and computation to the third-party cloud servers brings multifarious security and privacy challenges. In [25], an improved algorithm is proposed for secure outsourcing of matrix multiplication to minimize the execution burden without sacrificing the confidentiality and integrity of the inputs and 
outputs. The theory of algebraic hyperstructures has many application including cryptography. In [26], the authors propose the concept of fuzzy hyperlattice ordered group.

Transferring digital images over the network is susceptible to various types of attacks including cropping, JPEG compression, median filtering, Gaussian blur noise, rotation, and salt \& pepper noise. In [27], the researchers propose a method for digital image watermarking based on a combination of artificial neural networks and advanced encryption and decryption technique to provide copyright protection and security to digital bank cheque images. Wireless sensor networks are widely deployed in many applications for monitoring environments. A major concern in such networks is the secure and accurate communication of aggregated data. In [28], a cluster based method is proposed for confidentiality and integrity of concealed data aggregation in wireless sensor networks. The proposed method uses Elliptic Curve Cryptography-based Elgamal additive homomorphic encryption scheme for providing confidentiality and integrity. A nonce reuse/misuse resistance authentication encryption technique for better security bounds on modern TLS Cipher Suites and QUIC (Quick UDP Internet Communications) based Web Servers was used in [29]. Exploration of sensitive information retrieved from cloud databases is addressed in [30] where the authors present a homomorphic encryption approach that allows cloud servers to respond to queries while preserving privacy.

With the increasing popularity of speech recognition applications such as Alexa, Google Home, and Cortana, voice user interfaces (VUIs) are becoming common to interact with smartphones and consumer products. In [31] a prototype, known as GEORGIE, is used to achieve novel means of interaction inspired by the principles of hypnotherapy (a discrete interface ensuring that end-users' privacy is not compromised). Malicious software such as viruses automatically evolve to evade popular anti-malware scanners. Evolutionary algorithms are utilized in [32] to mutate the timid virus with a simple code evasion strategy to generate authentic variants of the malware. Distributed denial of service (DDoS) is one the notable threats in computer and network security during the past decade. It occurs when multiple machines generate massive amount of traffic to attach a specific target. In [33], a high scalable Spark streaming-based distributed and real-time DDoS detection system is proposed using k-means clustering algorithm. An improved method based on particle swan optimization to solve the tradeoff among the conflicting objectives in intrusion detection systems is descibed in [34].

Several articles in this special issue have addressed the design of optimal solutions for a variety of applications. In [35], the authors propose a multiobjective optimization model to reduce delay and energy consumption in vehicular fog computing (VFC). The results reveal superiority of the proposed VFC framework over generic cloud platforms. The approach in [36] applies unsupervised machine learning techniques based on fuzzy c-means++ clustering algorithm for designing routing protocol in wireless sensor networks with low power consumption. The growing number of nodes in wireless sensor networks require that architectures maintain low-power consumption per-node to comply with energy guaranty acceptable network lifetime. In [37], the authors propose to optimize the length of the mobile sink's traveling path to reduce the delay during data gathering in large-scale wireless sensor networks. An optimal data storage hosting model for multi-cloud environments using Sugeno-type fuzzy inference system that selects the appropriate storage type by considering the users' purpose and nature of usage in a cost-effective way was proposed in [39]. The study in [40] introduces a novel and efficient L-band log-periodic spiral antenna array design in radio interferometry for synthesis imaging.

An intelligent autonomous navigation system for unmanned aerial vehicles (UAV) in randomly changing complex environments is described in [41]. Road accidents in developing countries are increasing at an alarming rate. A case study presented in [42] develops a prediction model for road accidents in the State of Haryana, India using machine learning technique. Data mining and knowledge discovery has significant applications using medical and biological datasets. The study in [43] identifies an optimal subset of relevant features by integrating ReliefF and correlation feature selection techniques. Several optimization paradigms have been inspired from nature. A new modification of Moth-Flame Optimization is described in [44] by adding the concept of orthogonality in order to improve the performance for global optimization. Many complex real-world problems may be modeled using graphs. In [45], the authors present a link prediction model using topological feature extraction based on a geodesic distance measure. Another approach presented in [46] improves the performance of nature-inspired Spotted Hyena 
Optimizer through the combination of oppositional learning concept with mutation operator. In [47], the authors describe a new scalable parallel architecture to improve the performance of Galactic Swarm Optimization algorithm that was inspired from the motion of stars and galaxies under the influence of gravity. An application of meta-heuristic based Elitist Teaching Learning Based Optimization to nonlinear fractional order proportional integral derivative (PID) controller for tracking maximum power in photo-voltaic systems is presented in [48]. In [49], an improved multiverse optimization (IMVO) assisted global maximum power point tacking (MPPT) algorithm is presented for partially shaded photovoltaic system to obtain maximum power. A comprehensive review and classified list of more than 50 studies of strange behavior of nonlinear dynamic systems is presented in [50] to enable prevention of blackouts, disasters, and failures.

This special issue would not have been possible without the help of many participants. As guest editors, we take this opportunity to thank the authors for their contributions and the reviewers for their invaluable comments and timely responses. We would also like to thank the JIFS Editor-in-Chief and staff for their support during the preparation and production of this special issue.

\section{References}

[1] A.S. Ajeena Beegom and P. Chinmayan, Solving word sense disambiguation problem using combinatorial PSO, Journal of Intelligent \& Fuzzy Systems (JIFS), IOS Press, 2020.

[2] N. Akhtar, M.M. Sufyan Beg and M. Hussain, Extractive multi-document summarization using relative redundancy and coherence scores, Journal of Intelligent \& Fuzzy Systems (JIFS), IOS Press, 2020.

[3] E.-S.M. El-Alfy and S. Al-Azani, Empirical study on imbalanced learning of arabic sentiment polarity with neural word embedding, Journal of Intelligent \& Fuzzy Systems (JIFS), IOS Press, 2020.

[4] S. Sanagar, D. Gupta, Automated genre-based multi-domain sentiment lexicon adaptation using unlabeled data, Journal of Intelligent \& Fuzzy Systems (JIFS), IOS Press, 2020.

[5] N. Gupta, R. Singh and P. Bedi, Combining trust and reputation as user influence in cross domain group recommender system (CDGRS), Journal of Intelligent \& Fuzzy Systems (JIFS), IOS Press, 2020.

[6] P. Sujitha and S. Philomina, A computationally efficient method for human activity recognition based on spatio temporal cuboid and super normal vector, Journal of Intelligent \& Fuzzy Systems (JIFS), IOS Press, 2020.

[7] A. Alpana and S. Chand, An intelligent technique for the characterization of coal microscopic images using ensemble learning, Journal of Intelligent \& Fuzzy Systems (JIFS), IOS Press, 2020.
[8] S. Ramachandran, S. Kochitty, A. Vinekar and R. John, A fully convolutional neural network approach for the localization of optic disc in retinopathy of prematurity diagnosis, Journal of Intelligent \& Fuzzy Systems (JIFS), IOS Press, 2020.

[9] T.P. Shiji, T. Vinu, L. Rekha, S. Remya and P. Thara, Evolutionary intelligence for breast lesion detection in ultrasound images: A wavelet modulus maxima and SVM based approach, Journal of Intelligent \& Fuzzy Systems (JIFS), IOS Press, 2020.

[10] V. Dayanand, R. Gowtham, M. Senthilkumar, R.P. Sini and R. Sourav, A layered approach to detect elephants in live surveillance video streams using convolution neural networks, Journal of Intelligent \& Fuzzy Systems (JIFS), IOS Press, 2020.

[11] C. Jyotsna, J. Amudha, R. Raghavendra and N. Ravi, Intelligent gaze tracking approach for trail making test, Journal of Intelligent \& Fuzzy Systems (JIFS), IOS Press, 2020.

[12] J. Amudha, K.V. Divya and R. Aarthi, A fuzzy based system for target search using top-down visual attention, Journal of Intelligent \& Fuzzy Systems (JIFS), IOS Press, 2020.

[13] Y. Navdeep, S. Vijander; R. Asha and G. Sonal, An improved hyper smoothing function based edge detection algorithm for noisy images, Journal of Intelligent \& Fuzzy Systems (JIFS), IOS Press, 2020.

[14] S. Lalitha, S. Jahnavi and S. Supraja, A vital neurodegenerative disorder detection using speech cues, Journal of Intelligent \& Fuzzy Systems (JIFS), IOS Press, 2020.

[15] S. Utkarsh, G. Akshay, B. Dipjyoti and A. Wasim, Foreign accent classification using deep neural nets, Journal of Intelligent \& Fuzzy Systems (JIFS), IOS Press, 2020.

[16] M.T. Vyshnav, K.S. Sachin, M. Neethu and K.P. Soman, Random fourier feature based music-speech classification, Journal of Intelligent \& Fuzzy Systems (JIFS), IOS Press, 2020.

[17] S. Hemendra, P. Devashri, R.E. Damodar, B. Annushree and M. Malkauthekar, Brain computer interface for measuring the impact of yoga on concentration levels in engineering students, Journal of Intelligent \& Fuzzy Systems (JIFS), IOS Press, 2020.

[18] D.K. Manu and P. Karthik, Enhancement of hydrophone sensitivity by varying mandrel parameters for detection of acoustic waves in underwater environment, Journal of Intelligent \& Fuzzy Systems (JIFS), IOS Press, 2020.

[19] C.C.K. Dodla and T. Shikha, Hybrid architecture for multiple transforms for signal processing applications, Journal of Intelligent \& Fuzzy Systems (JIFS), IOS Press, 2020.

[20] R.K. Remya and M. Wilscy, Image forgery detection using deep textural features from local binary pattern map, Journal of Intelligent \& Fuzzy Systems (JIFS), IOS Press, 2020.

[21] R. Anushiadevi, P. Padmapriya, R.J. Balaguru and A. Rengarajan, A high payload separable reversible data hiding in cipher image with good decipher image quality, Journal of Intelligent \& Fuzzy Systems (JIFS), IOS Press, 2020.

[22] S. Santhu, S. Trivandrum, P.J. Anna, G. Chemmannur and N. Madhu, Tongue print identification using deep CNN for forensic analysis, Journal of Intelligent \& Fuzzy Systems (JIFS), IOS Press, 2020.

[23] A.M. Jitendra and S. Poonam, Occluded face recognition using noncoherent dictionary, Journal of Intelligent \& Fuzzy Systems (JIFS), IOS Press, 2020.

[24] R.R. Ashoka and P. Kumaran, Multi-biometric cryptosystem using graph for secure cloud authentication, Journal of Intelligent \& Fuzzy Systems (JIFS), IOS Press, 2020. 
[25] K. Malay, M. Vaibhav, S. Anurag, S. Munendra and V. Manu, A novel publicly delegable secure outsourcing algorithm for large-scale matrix multiplication, Journal of Intelligent \& Fuzzy Systems (JIFS), IOS Press, 2020.

[26] D. Preethi, J. Vimala, D. Bijan and S. Rajareega, Biological inheritance on fuzzy hyperlattice ordered group, Journal of Intelligent \& Fuzzy Systems (JIFS), IOS Press, 2020.

[27] S.G. Sudhanshu, Combination of neural network and advanced encryption and decryption technique is used for digital image watermarking, Journal of Intelligent \& Fuzzy Systems (JIFS), IOS Press, 2020.

[28] P.T. Suriya, V. Meena, K. Prachetha, B. Aravind, K.C.S. Bharathkumar and T. Sethukarasi, Energy measure cluster based concealed aggregation for confidentiality and integrity in wsn, Journal of Intelligent \& Fuzzy Systems (JIFS), IOS Press, 2020.

[29] B. Arunkumar and G. Kousalya, Nonce reuse/misuse resistance authentication encryption schemes for modern tls cipher suites and quic based web servers, Journal of Intelligent \& Fuzzy Systems (JIFS), IOS Press, 2020.

[30] S.R. Jitendra and S.V. Kunwar, Homomorphic encryption approach for exploration of sensitive information retrieval, Journal of Intelligent \& Fuzzy Systems (JIFS), IOS Press, 2020.

[31] C. Anirban and R. Rithvik, Hypnotic computer interface (hypCI) using georgie: An approach to design discrete voice user interfaces, Journal of Intelligent \& Fuzzy Systems (JIFS), IOS Press, 2020.

[32] M. Ritwik and C.S. Velayutham, A preliminary investigation into automatically evolving computer viruses using evolutionary algorithms, Journal of Intelligent \& Fuzzy Systems (JIFS), IOS Press, 2020.

[33] V.P. Nilesh, C.R. Krishna, K. Krishan, S-ddos: Apache spark based real-time ddos detection system, Journal of Intelligent \& Fuzzy Systems (JIFS), IOS Press, 2020.

[34] D. Anne and T. Ciza, Improved PSO for optimizing the performance of intrusion detection systems, Journal of Intelligent \& Fuzzy Systems (JIFS), IOS Press, 2020.

[35] M. Hussain, M.S. Alam, N. Akhtar and M.M. Sufyan Beg, Towards minimizing delay and energy consumption in vehicular fog computing (vfc), Journal of Intelligent \& Fuzzy Systems (JIFS), IOS Press, 2020.

[36] S.C. Veervrat, T. Rajiv and P. Rahul, An intelligent low power consumption routing protocol to extend the lifetime of wireless sensor networks based on fuzzy c-means++ clustering algorithm, Journal of Intelligent \& Fuzzy Systems (JIFS), IOS Press, 2020.

[37] P.T. Suriya, T. Sethukarasi and V. Veeramuthu, Krill herd based TSP approach for mobile sink path optimization in large scale wireless sensor networks, Journal of Intelligent \& Fuzzy Systems (JIFS), IOS Press, 2020.
[38] M. Senthilkumar, J. Eric, M.S. Advaith, K.S. Gopikrishna and G. Rohit, A flattened architecture for distributed mobility management in IPV6 networks, Journal of Intelligent \& Fuzzy Systems (JIFS), IOS Press, 2020.

[39] M. Sujatha, K. Geetha, P. Balakrishnan and T. Renugadevi, An optimal data storage hosting model using sugeno-type fuzzy inference system for multi-cloud environments, Journal of Intelligent \& Fuzzy Systems (JIFS), IOS Press, 2020.

[40] K. Pradeep, T. Rajagopalan and G.A.S. Sundaram, A logperiodic spiral antenna array for l-band radio interferometric imaging, Journal of Intelligent \& Fuzzy Systems (JIFS), IOS Press, 2020.

[41] Z. Anton, C. Sergei, S. Sergei and N. Anatoliy, Intelligent autonomous navigation system for UAV in randomly changing environmental conditions, Journal of Intelligent \& Fuzzy Systems (JIFS), IOS Press, 2020.

[42] M. Navdeep, S. Hemant and G. Tripta, Application of machine-learning technique for prediction of road accidents in state of haryana-a novel approach, Journal of Intelligent \& Fuzzy Systems (JIFS), IOS Press, 2020.

[43] S. Alok, K.P. Sanjeev, G. Srishti, B. Reddy and T. Diwakar, Knowledge discovery in medical and biological datasets by integration of relief-F and correlation feature selection techniques, Journal of Intelligent \& Fuzzy Systems (JIFS), IOS Press, 2020.

[44] P. Rosy, K.M. Santosh and J. Jemarani, An orthogonal moth flame optimization for global optimization and application to model order reduction problem, Journal of Intelligent \& Fuzzy Systems (JIFS), IOS Press, 2020.

[45] J.D. Salam and S. Buddha, Link prediction model based on geodesic distance measure using various machine learning classification models, Journal of Intelligent \& Fuzzy Systems (JIFS), IOS Press, 2020.

[46] P. Nibedan, K.M. Santosh, S. Sarishma and K. Abhirup, Oppositional spotted hyena optimizer with mutation operator for global optimization and application in training wavelet neural network, Journal of Intelligent \& Fuzzy Systems (JIFS), IOS Press, 2020.

[47] D.A. Phadke, K.S. Umadevi, B.P.S. Bhardwaj and G.B. Amali, A new parallel galactic swarm optimization algorithm for training artificial neural networks, Journal of Intelligent \& Fuzzy Systems (JIFS), IOS Press, 2020.

[48] R. Anuj, K.J. Shyama, K. Bhavnesh and M. Vijay, Nonlinear fractional order PID controller for tracking maximum power in photo-voltaic system, Journal of Intelligent \& Fuzzy Systems (JIFS), IOS Press, 2020.

[49] C. Urvashi, S. Vijander, K. Bhavnesh and R. Asha, An improved MVO assisted global MPPT algorithm for partially shaded PV system, Journal of Intelligent \& Fuzzy Systems (JIFS), IOS Press, 2020.

[50] N. Sunil and J. Kantilal, Trends in chaos and instability for understanding system complexity, Journal of Intelligent \& Fuzzy Systems (JIFS), IOS Press, 2020. 